\title{
Phase-field simulation of hydraulic fracturing with a revised fluid model and hybrid solver
}

\author{
Bin Chen ${ }^{\mathrm{a}, \mathrm{b}}$, Yanan Sun ${ }^{\mathrm{a}}$, Beatriz R. Barboza ${ }^{\mathrm{a}}$, Andrew R Barron ${ }^{\mathrm{c}}$, Chenfeng Li ${ }^{\mathrm{a}, \mathrm{c}, 1,}$ \\ ${ }^{a}$ Zienkiewicz Centre for Computational Engineering, College of Engineering, Swansea University Bay Campus, Swansea SA1 8EN, United \\ Kingdom \\ ${ }^{b}$ State Key Laboratory of Coal Resources and Safe Mining, China University of Mining and Technology, Xuzhou, 221116, China \\ ${ }^{c}$ Energy Safety Research Institute, College of Engineering, Swansea University Bay Campus, Swansea SA1 8EN, United Kingdom
}

\begin{abstract}
With an intrinsic advantage in describing complex fracture networks, the phase field method has demonstrated promising potential for the simulation of hydraulic fracturing processes in recent literatures. We critically examine the existing phase-field hydraulic fracturing models, and propose a hybrid solution scheme with a revised fluid model. Specifically, the formation deformation and phase field are solved using the finite element method (FEM), while the fluid flows are solved using the finite volume method (FVM). The proposed hybrid scheme is validated with the analytical solution for the toughness-dominated fracture propagation and is tested on the complex hydraulic fracturing process in a naturally fractured formation. Demonstrated by numerical examples, the proposed hybrid phase-field framework has several advantages: 1) it captures the effect of fluid pressure inside the fracture and reservoir more accurately than existing models; 2) it provides a sharper capture of formation fractures; 3 ) it avoids the nonphysical oscillation of fluid pressure when using a pure FEM solver; and 4) it has a superior performance in mesh and time step convergence.
\end{abstract}

Keywords: Phase field method; Fluid-driven fracture; Porous media; Finite element method; Finite volume method; Discrete fracture network

\section{Introduction}

Fluid-driven fractures are common in geotechnical, environmental and petroleum engineering, among which a typical case is the hydraulic fracturing process in the oil and gas industry. Hydraulic fracturing plays an important role in increasing the reservoir permeability and hence enhancing oil and gas recovery. It is a coupled physical process involving porous media deformation, fracture propagation and fluid flow inside fracture and reservoir formation [1]. The process is further complicated by the presence of natural fracture network and the heterogeneity and anisotropy of the formation. Hydraulic fracturing, owing to its importance and complexity, has been extensively studied by both academia and industries.

During the past decades, diverse numerical approaches have been adapted and improved to simulate the hydraulic fracturing process. Continuum based methods treat the formation as a continuum, with the fractures modelled as discrete or smeared cracks. Using the discrete fracture models, the initiation and propagation of fractures are simulated by inserting discontinuities, and the associated numerical methods include the finite element method (FEM) [2, 3, 4] boundary element method (BEM) [5] and extended finite element method (XFEM) [6, 7, 8]. As the fracture surfaces need to be continuously tracked, the discrete fracture models often require remeshing and become impractical if

\footnotetext{
${ }^{1}$ Corresponding author. E-mail: c.f.li@swansea.ac.uk
} 

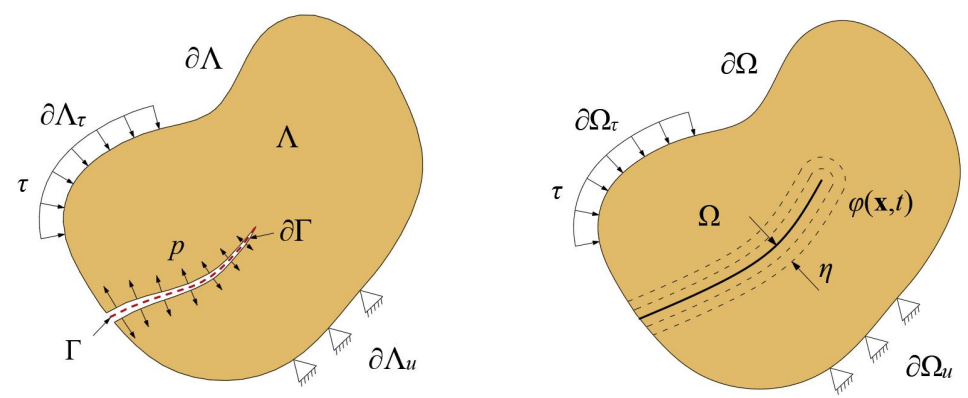

Figure 1. Comparison between the classic discrete fracture model (left) and the phase-field fracture model (right)

complex fracture networks are present. Using the smeared fracture models, the fractures are represented by damaged elements and the damage of the elements is normally governed by a fracture criterion such as Mohr-coulomb criterion $[9,10]$. Since the topology of spatial discretization does not change in the smeared fracture models, remeshing is avoided, but the computed fracture path is smeared. Unlike the continuum based methods, the non-continuum based methods describe the material with discrete particles (blocks) and bonds (joints), and the initiation and propagation of fractures are simulated by the breakage of internal bonds or joints. The non-continuum based methods do have advantages in dealing with complex fractures, but the simulated fracture paths are often dependent on the specific discretization and difficult to achieve convergence. Hybrid methods such as the finite-discrete element method (FDEM) have also been developed to simulate hydraulic fracturing with complex fracture propagations $[11,12,13]$.

A promising approach to cope with complex fracture propagation is the phase field method, which has been used in fracture simulation since 1998 [14, 15]. The phase field method introduces an extra parameter, i.e. the phase field, that varies continuously from 0 to 1 and represents all fractures in the simulation domain. Compared with the traditional discrete fracture models based on FEM, BEM or XFEM representations, the phase-field fracture model has several attractive features. First, the fracture initiation, propagation, branching and merging are solved in a unified framework and no extra criteria or numerical treatment is needed. Secondly, it treats multiple fractures simultaneously as an energy minimization problem, avoiding the tedious tracking and assessing of each individual fracture propagation. Thirdly, it can easily account for material heterogeneity. The main drawback of the phase field method is its high computational cost, in that a finer mesh is usually required for accurate solution.

Applying the phase field method to fluid-driven fractures is however a more recent advancement. First reported in [16], several phase field models have been presented to simulate the hydraulic fracturing process, using different approaches to combine the effect of fluid flow into the classic phase-field fracture model. The first approach considers the fluid effect as part of the work done by external forces $[16,17,18]$. The second approach combines the fluid potential energy into the total energy, and solves the displacement, pressure and phase field via the variational method [19]. The third approach simulates the fluid transport and rock deformation in a volume fraction based model [20]. However, the relationship between different models remains unclear, and no general consensus is reached yet.

The present study has two objectives: 1) to clarify the difference and relationship between various fluid models in phase-field simulation of hydraulic fracturing and to propose a new fluid model with higher accuracy; and 2) to develop an FE-FV solution scheme for phase-field hydraulic fracturing simulation and to avoid the various numerical issues encountered in existing approaches. The rest of the paper is organized as follows. $\S 2$ provides a brief overview of existing phase-field hydraulic fracturing models. $\S 3$ presents a new FE-FV solution scheme with a revised fluid model. § 4 compares different fluid models in phase-field hydraulic fracturing simulation, validates the accuracy of the proposed model, and demonstrates the advantages of the proposed scheme in a range of numerical cases. Concluding remarks are made in $\S 5$.

\section{Phase field models for hydraulic fracturing}

\subsection{Phase-field representation of fracture}

The classic discrete fracture model and the phase-field fracture model are compared in Figure 1. For the classic discrete fracture model, the material deformation is first solved using a numerical method like FEM, then the fracture 
propagation is determined by the strain and stress state of material near the crack tip, and finally the material discretization is updated by inserting discontinuities. The phase field model treats the material deformation and fracture propagation as a unified energy minimization problem, and they are solved together following a variational framework. To do so, the phase field variable is introduced to represent the fracture evolution and a fracture surface energy is incorporated into the total energy. A phase-field fracture model is generally expressed as [18, 21]:

$$
[\hat{\mathbf{u}}, \hat{\varphi}]=\operatorname{Arg}\{\inf E(\mathbf{u}, \varphi)\}
$$

where $\mathbf{u}$ is the displacement of material, $\varphi$ is the phase field representing fracture, and $E$ is the total energy as a function of $\mathbf{u}$ and $\varphi$. The phase field $\varphi$ is real-valued between 0 and 1 , with the value 0 at the fracture and the value 1 further away from the fracture. The total energy of a linear elastic medium containing fractures is expressed as:

$$
E=\Psi_{e}+\Psi_{\text {frac }}
$$

where $\Psi_{e}$ and $\Psi_{\text {frac }}$ denote the elastic energy and the fracture surface energy respectively. The fracture surface energy is expressed as:

$$
\Psi_{\text {frac }}=G_{c} \mathcal{H}^{N-1}(\Gamma)
$$

where $G_{c}$ is the energy release rate, and $\mathcal{H}^{N-1}(\Gamma)$ is the N-1-dimensional Hausdorff measure of fracture path $\Gamma$, expressed as

$$
\mathcal{H}^{N-1}=\int_{\Omega}\left(\frac{(1-\varphi)^{2}}{2 \eta}+\frac{\eta}{2}|\nabla \varphi|^{2}\right) d \Omega
$$

where $\Omega$ denotes the simulation domain in the phase field model and $\eta$ is the length scale parameter. When $\eta$ approaches 0 , the diffusive crack represented by the phase field $\varphi$ approximates the sharp crack solved with the discrete fracture approach. The elastic strain energy in Eqn. (2) is defined as:

$$
\Psi_{e}=\int_{\Omega} \psi_{e}(\boldsymbol{\varepsilon}, \varphi) d \Omega-\int_{\partial \Omega} \boldsymbol{\tau} \cdot \mathbf{u} d s-\int_{\Omega} \varphi \rho \boldsymbol{g} \cdot \mathbf{u} d \Omega
$$

where $\tau$ is the external surface force, $\rho$ the density of material, and $\boldsymbol{g}$ the gravity acceleration. $\psi_{e}(\boldsymbol{\varepsilon}, \varphi)$ is the elastic energy density function expressed as:

$$
\psi_{e}(\boldsymbol{\varepsilon}, \varphi)=\frac{1}{2} \varphi^{2} \boldsymbol{\varepsilon}:(\mathbb{C}: \boldsymbol{\varepsilon})
$$

where $\mathbb{C}$ is the elastic tensor and $\boldsymbol{\varepsilon}$ denotes the strain. However the above isotropic formulation can lead to fictitious fractures due to compression [22]. In order to avoid this, two split strategies [22, 23] have been proposed to compute the elastic strain energy as a combination of tensile (denoted by superscript ${ }^{+}$or subscript $_{+}$) and compressive (denoted by superscript ${ }^{-}$or subscript $\_$) strain energies. According to Miehe's formation [23], the elastic energy density is defined as:

$$
\psi_{e}(\boldsymbol{\varepsilon}, \varphi)=\psi_{e}^{-}(\boldsymbol{\varepsilon}, \varphi)+\varphi^{2} \psi_{e}^{+}(\boldsymbol{\varepsilon}, \varphi)
$$

where the tensile and compressive parts of the elastic energy density are expressed as:

$$
\begin{aligned}
& \psi_{e}^{+}(\boldsymbol{\varepsilon}, \varphi)=\lambda\langle\operatorname{tr}[\varepsilon]\rangle_{+}^{2} / 2+\mu \operatorname{tr}\left[\varepsilon_{+}^{2}\right] \\
& \psi_{e}^{-}(\boldsymbol{\varepsilon}, \varphi)=\lambda\langle\operatorname{tr}[\varepsilon]\rangle_{-}^{2} / 2+\mu \operatorname{tr}\left[\varepsilon_{-}^{2}\right]
\end{aligned}
$$

where $\lambda$ and $\mu$ are the Lamé's first parameter and shear modulus respectively, $\boldsymbol{\varepsilon}_{+}=\sum_{i=1}^{\delta}\left\langle\varepsilon^{i}\right\rangle_{+} \boldsymbol{n}^{i} \otimes \boldsymbol{n}^{i}$ and $\boldsymbol{\varepsilon}_{-}=$ $\sum_{i=1}^{\delta}\left\langle\varepsilon^{i}\right\rangle_{-} \boldsymbol{n}^{i} \otimes \boldsymbol{n}^{i} . \varepsilon^{i}$ and $\boldsymbol{n}^{i}$ are the principal strains and corresponding directions, $i$ is from 1 to $\delta, \delta=2$ in $2 \mathrm{D}$ and 3 in $3 \mathrm{D}$. The bracket operators are defined as $\langle x\rangle_{+}=(|x|+x) / 2$ and $\langle x\rangle_{-}=(|x|-x) / 2$. The corresponding stress is expressed as:

$$
\boldsymbol{\sigma}=\varphi^{2}\left[\lambda\langle\operatorname{tr}(\boldsymbol{\varepsilon})\rangle_{+} \mathbf{I}+2 \mu \boldsymbol{\varepsilon}_{+}\right]+\left[\lambda\langle\operatorname{tr} \boldsymbol{\varepsilon}\rangle_{-} \mathbf{I}+2 \mu \boldsymbol{\varepsilon}_{-}\right]
$$

\subsection{Existing fluid models in phase-field simulation of hydraulic fracturing}

The phase field method was first applied to hydraulic fracturing simulation by Bourdin et al. [16], and since then several different models have been developed to integrate the effect of fluid pressure along the fracture and the pore pressure in the formation into the classic phase-field fracture model. 


\subsubsection{Modelling fluid effects as work by external forces}

A typical approach to incorporate the fluid effect into the phase-field fracture model is through the work by external forces. Taking into account the fluid effect, the elastic energy $\Psi_{e}$ can be modified as [16]

$$
\Psi_{e}=\int_{\Omega} \psi_{e}(\boldsymbol{\varepsilon}, \varphi) d \Omega-\int_{\partial \Omega} \boldsymbol{\tau} \cdot \mathbf{u} d s-\int_{\Omega} \varphi \rho \boldsymbol{g} \cdot \mathbf{u} d \Omega-W_{\text {fluid }}
$$

In the above equation, the work done by the fluid pressure inside the fracture is expressed as:

$$
W_{\text {fluid }}=\int_{\Gamma} p\left(\mathbf{u}^{+}-\mathbf{u}^{-}\right) \cdot \mathbf{n} d s \approx \int_{\Omega} p \mathbf{u} \cdot \nabla \varphi d \Omega
$$

where $p$ is the fluid pressure, $\mathbf{u}^{+}$and $\mathbf{u}^{-}$are the displacement at two sides of the fracture, $\mathbf{u}$ is the displacement field, and $\mathbf{n}$ is the oriented normal direction to the fracture path $\Gamma$.

More recently, the above model was extended to account for the effect of pore pressure [17], and the elastic strain energy becomes:

$$
\Psi_{e}=\frac{1}{2} \int_{\Omega} \mathbb{C}\left(\varphi \boldsymbol{\varepsilon}-\frac{\alpha}{3 \kappa} p \mathbf{I}\right):\left(\varphi \boldsymbol{\varepsilon}-\frac{\alpha}{3 \kappa} p \mathbf{I}\right) d \Omega-\int_{\partial \Omega} \boldsymbol{\tau} \cdot \mathbf{u} d s-\int_{\Omega} \boldsymbol{f} \cdot \mathbf{u} d \Omega-\int_{\Omega} p \mathbf{u} \cdot \nabla \varphi d \Omega
$$

where $\alpha$ is the Biot's coefficient and $f$ is the body force. In this equation, the equivalent work done by the fluid can be expressed as:

$$
W_{\text {fluid }}=\int_{\Omega} \varphi^{2} \alpha p \nabla \cdot \mathbf{u} d \Omega+\int_{\Omega} p \mathbf{u} \cdot \nabla \varphi d \Omega
$$

Adopted in $[24,25,26]$, another approach to compute the work done by the fluid pressure inside the fracture is expressed as

$$
-\int_{\Gamma} p \mathbf{n} \cdot \mathbf{u} d s=-\int_{\Lambda} \nabla \cdot(p \mathbf{u}) d \Lambda+\int_{\partial \Lambda} p \mathbf{u} \cdot \mathbf{n} d s=-\int_{\Lambda}(\mathbf{u} \cdot \nabla p+p \nabla \cdot \mathbf{u}) d \Lambda+\int_{\partial \Lambda} p \mathbf{u} \cdot \mathbf{n} d s
$$

where $\mathbf{n}$ is the oriented normal direction to the fracture path $\Gamma$. $\Gamma$ and $\Lambda$ are the fracture path and simulation domain respectively in the classic discrete fracture model, as shown in Figure 1. The last term vanishes with the Dirichlet boundary condition. Using the phase field approximation

$$
-\int_{\Lambda}(\mathbf{u} \cdot \nabla p+p \nabla \cdot \mathbf{u}) d \Lambda \approx-\int_{\Omega} \varphi^{2}(\mathbf{u} \cdot \nabla p+p \nabla \cdot \mathbf{u}) d \Omega
$$

The work done by the pore pressure is expressed as:

$$
\int_{\Lambda} \alpha p \nabla \cdot \mathbf{u} d \Lambda \approx \int_{\Omega} \varphi^{2} \alpha p \nabla \cdot \mathbf{u} d \Omega
$$

Therefore, the potential energy due to the fluid flow is

$$
W_{\text {fluid }}=\int_{\Omega}(\alpha-1) \varphi^{2} p \nabla \cdot \mathbf{u} d \Omega-\int_{\Omega} \varphi^{2} \mathbf{u} \cdot \nabla p d \Omega
$$

\subsubsection{Modelling fluid effects as the fluid potential energy}

The fluid effect has also been modelled by introducing a potential energy for fluid into the total energy [27, 28, 19]

$$
E=\Psi_{e}+\Psi_{\text {fluid }}+\Psi_{\text {frac }}
$$


where $\Psi_{\text {fluid }}$ is the potential energy density for fluid and satisfies [19]

$$
\frac{\partial \Psi_{\text {fluid }}}{\partial \mathbf{u}}=-\alpha p \frac{\partial(\nabla \cdot \mathbf{u})}{\partial \mathbf{u}}
$$

Therefore, the equivalent work done by the fluid pressure $W_{\text {fluid }}$ in Enq. (11) can be expressed as

$$
W_{\text {fluid }}=\int_{\Omega} \alpha p \nabla \cdot \mathbf{u} d \Omega
$$

In the potential energy approach, the work done by the fluid pressure inside the fracture and the work done by the pore pressure are not distinguished.

\subsubsection{Other models}

In the aforementioned phase field models for hydraulic fracturing, the fluid effect is represented by specific terms derived from the work done by the fluid pressure or the fluid potential energy. Different from these models, [20] introduced the concept of volume fraction into the phase field models for hydraulic fracturing. Solid deformation and fluid transport are represented by the corresponding volume fractions and velocities, which are solved with a continuity equation and two momentum equations. As this volume-fraction framework is independent from the external work and potential energy approaches, the comparison between this model and aforementioned models is not considered in this work.

\section{A new FE-FV scheme for phase-field hydraulic fracturing simulation}

\subsection{Governing equations}

In this paper, we propose a new formulation of the total energy:

$$
\begin{gathered}
E=\Psi_{e}+\Psi_{\text {frac }} \\
\Psi_{e}=\int_{\Omega} \psi_{e}(\boldsymbol{\varepsilon}, \varphi) d \Omega+\int_{\partial \Omega} \tau \cdot \mathbf{u} d s-W_{\text {fluid }} \\
W_{\text {fluid }}=\int_{\Omega}\left[1-(1-\varphi)^{2}\right] \alpha p \nabla \cdot \mathbf{u} d \Omega+\int_{\Omega}(1-\varphi)^{2} p \nabla \cdot \mathbf{u} d \Omega \\
\Psi_{\text {frac }}=G_{c} \int_{\Omega} \frac{(1-\varphi)^{2}}{2 \eta}+\frac{\eta}{2}|\nabla \varphi|^{2} d \Omega
\end{gathered}
$$

where the strain energy density $\psi_{e}(\boldsymbol{\varepsilon}, \varphi)$ is defined according to the Miehe's energy split strategy (7) to avoid the degradation due to compression. In the above expression, a new formulation of the fluid potential is proposed, where the matrix and fracture domains are defined by the functions $\left[1-(1-\varphi)^{2}\right]$ and $(1-\varphi)^{2}$, respectively. The proposed model degrades to Miehe's model for $\alpha=1$.

Applying the variational principle $\delta E / \delta \mathbf{u}=0$ and $\delta E / \delta \varphi=0$ leads to

$$
\begin{gathered}
\int_{\Omega} \sigma: \delta \boldsymbol{\varepsilon}-\left[1-(1-\varphi)^{2}\right] \alpha p \frac{\partial(\nabla \cdot \mathbf{u})}{\partial \mathbf{u}} \cdot \delta \mathbf{u} d \Omega-\int_{\Omega}(1-\varphi)^{2} p \frac{\partial(\nabla \cdot \mathbf{u})}{\partial \mathbf{u}} \cdot \delta \mathbf{u} d \Omega+\int_{\partial \Omega} \boldsymbol{\tau} \cdot \delta \mathbf{u} d s=0 \\
\int_{\Omega} 2 \varphi \psi^{+}(\boldsymbol{\varepsilon})+2(\varphi-1) \alpha p \nabla \cdot \mathbf{u} \delta \varphi d \Omega+G_{c} \int_{\Omega} \frac{(\varphi-1)}{\eta} \delta \varphi+\eta \nabla \varphi \cdot \nabla \delta \varphi d \Omega-\int_{\Omega} 2(\varphi-1) \delta \varphi p \nabla \cdot \mathbf{u} d \Omega=0
\end{gathered}
$$


Instead of simulating the fluid flow in the fracture and matrix in separate domains as in $[24,25,26]$, a continuity equation with a generic permeability for the fluid flow over the whole domain is adopted here [27, 29, 30]

$$
\left(\alpha\left[1-(1-\varphi)^{2}\right]+(1-\varphi)^{2}\right) \frac{\partial \varepsilon_{v}}{\partial t}+\nabla \cdot\left(\mathbf{k}_{p e r}(\nabla p-\rho g \nabla z)\right)=Q-\frac{1}{M} \frac{\partial p}{\partial t}
$$

where $\varepsilon_{v}$ is the volume strain, $M$ is the Biot's modulus, $Q$ is the fluid injection flow rate and the permeability tensor $\mathbf{k}_{p e r}$ is defined as

$$
\mathbf{k}_{p e r}=\frac{k_{r}}{\mu} \mathbf{I}+(1-\varphi)^{\epsilon} \mathbf{k}_{f}
$$

where the first term is the isotropic Darcy permeability and the second term describes an additional permeability tensor due to fracture. $\epsilon$ is an additional parameter to restrict the increase of fracture permeability in a small area very close to the fracture and is set to 50 here according to [19]. The $\mathbf{k}_{f}$ is defined as

$$
\mathbf{k}_{f}=\left(\frac{w^{3}}{12 \mu h}-\frac{k_{r}}{\mu}\right)(\mathbf{I}-\mathbf{n} \otimes \mathbf{n})
$$

where $h$ is the mesh size, $\mathbf{n}$ is the oriented normal direction to the fracture path and is defined by the direction of maximum principal stress. The fracture width is computed directly from the maximum principle strain and mesh size.

\subsection{Temporal and spatial discretization}

According to Eqn. (26), Eqn. (27) and Eqn. (28), rock deformation is computed based on quasi-static assumption and the transient term only exists in fluid continuity equations. The backward Euler method is adopted to discretize the equation on the temporal dimension. With respect to the spatial discretization, the FEM is adopted to solve the displacement and phase field and both the FEM and FVM are implemented to solve the fluid flows over the whole domain.

\subsubsection{Displacement and phase field discretization}

The displacement and phase field are discretized with the FEM:

$$
\mathbf{u}=\sum_{I=1}^{m} \mathbf{N}_{I}^{u} \mathbf{u}_{I}, \varphi=\sum_{I=1}^{m} N_{I} \varphi_{I}, \mathbf{N}_{I}^{u}=\left[\begin{array}{cc}
N_{I} & 0 \\
0 & N_{I}
\end{array}\right]
$$

where $N_{I}, \mathbf{u}_{I}$ and I are the shape function, displacement and phase field parameter at node I, respectively. The strain and the gradient of phase field are expressed as

$$
\boldsymbol{\varepsilon}=\sum_{I=1}^{m} \mathbf{B}_{I}^{u} \mathbf{u}_{I}, \nabla \varphi=\sum_{I=1}^{m} \mathbf{B}_{I} \varphi_{I}
$$

where

$$
\boldsymbol{\varepsilon}=\left[\begin{array}{c}
\varepsilon_{11} \\
\varepsilon_{22} \\
\gamma_{12}
\end{array}\right]=\left[\begin{array}{c}
\varepsilon_{11} \\
\varepsilon_{22} \\
2 \varepsilon_{12}
\end{array}\right], \mathbf{B}_{I}^{u}=\left[\begin{array}{cc}
N_{1, x} & 0 \\
0 & N_{1, y} \\
N_{1, y} & N_{1, x}
\end{array}\right], \mathbf{B}_{I}=\left[\begin{array}{c}
N_{1, x} \\
N_{1, y}
\end{array}\right]
$$

Test functions and their derivatives are expressed as

$$
\begin{aligned}
\delta \mathbf{u} & =\sum_{I=1}^{m} N_{I} \delta \mathbf{u}_{I}, \delta \varphi=\sum_{I=1}^{m} N_{I} \delta \varphi_{I} \\
\delta \boldsymbol{\varepsilon} & =\sum_{I=1}^{m} \mathbf{B}_{I}^{u} \delta \mathbf{u}_{I}, \nabla \delta \varphi=\sum_{I=1}^{m} \mathbf{B}_{I} \delta \varphi_{I}
\end{aligned}
$$

Combining the above equations, the FE discretization for the displacement and phase field can be summarized as:

$$
\left\{\begin{array}{l}
\mathbf{K}_{u u} \mathbf{u}+\mathbf{K}_{u p} \mathbf{p}=\mathbf{F}_{u} \\
\mathbf{K}_{\varphi \varphi} \boldsymbol{\varphi}=\mathbf{F}_{\varphi}
\end{array}\right.
$$




$$
\begin{gathered}
\text { Author } / X X(2020) 00-19 \\
\mathbf{K}_{u u}=\int_{\Omega} \mathbf{B}^{u T} \mathbf{D} \mathbf{B}^{u} d \Omega \\
\mathbf{K}_{\varphi \varphi}=\int_{\Omega} G_{c} \eta \mathbf{B}^{T} \mathbf{B} d x+\int_{\Omega}\left(2(\psi(\varepsilon)+\alpha p \nabla \cdot \mathbf{u}-p \nabla \cdot \mathbf{u})+\frac{G_{c}}{\eta}\right) \mathbf{N}^{\varphi T} \mathbf{N}^{\varphi} d \Omega \\
\mathbf{F}_{\varphi}=G_{c} \int_{\Omega} \frac{1}{\eta} N^{\varphi} d x+\int_{\Omega} 2(\alpha-1) p \nabla \cdot \mathbf{u} \delta \varphi d \Omega \\
\mathbf{K}_{u p}=\left\{\begin{array}{l}
\int_{\Omega}\left(\alpha\left[1-(1-\varphi)^{2}\right]+(1-\varphi)^{2}\right)\left[\mathbf{B}^{u}(1,:)+\mathbf{B}^{u}(2,:)\right]^{T} \delta p d \Omega \quad \text { for FEM-discretized pressure } \\
-\int_{\Omega}\left(\alpha\left[1-(1-\varphi)^{2}\right]+(1-\varphi)^{2}\right)\left[\mathbf{B}^{u}(1,:)+\mathbf{B}^{u}(2,:)\right]^{T} d \Omega \quad \text { for FVM-discretized pressure }
\end{array}\right.
\end{gathered}
$$

where $\mathbf{D}$ is the elastic stiffness matrix determined according to Miehe's stress splitting (Eqn. (10)).

\subsubsection{Fluid pressure discretization}

Following the FEM approach, the fluid continuity equation can be discretized as:

$$
\mathbf{K}_{p u}\left(\mathbf{u}-\mathbf{u}_{n}\right)+\mathbf{K}_{p p}^{(1)} \mathbf{p}+\mathbf{K}_{p p}^{(2)}\left(\mathbf{p}-\mathbf{p}_{n}\right)=\mathbf{F}_{p}
$$

where

$$
\mathbf{K}_{p u}=\int_{\Omega}\left(\alpha\left[1-(1-\varphi)^{2}\right]+(1-\varphi)^{2}\right) \delta p\left[\mathbf{B}^{u}(1,:)+\mathbf{B}^{u}(2,:)\right] d \Omega
$$

Therefore $\mathbf{K}_{p u}=-\mathbf{K}_{u p}^{T}$

$$
\begin{gathered}
\mathbf{K}_{p p}^{(1)}=\int_{\Omega} \mathbf{B}^{T} \mathbf{k}_{p e r} \mathbf{B} d \Omega \\
\mathbf{K}_{p p}^{(2)}=\int_{\Omega} \frac{1}{M} \mathbf{N}^{T} \mathbf{N} d \Omega \\
\mathbf{F}_{p}=\mathbf{Q} d t
\end{gathered}
$$

Following the FVM approach, the governing equation of the fluid flow becomes:

$$
\mathbf{K}_{p u}\left(\mathbf{u}-\mathbf{u}_{n}\right)+\mathbf{K}_{p p}^{(1)} \mathbf{p}+\mathbf{K}_{p p}^{(2)}\left(\mathbf{p}-\mathbf{p}_{n}\right)=\mathbf{F}_{p}
$$

where $\mathbf{K}_{p p}^{(1)}$ is obtained through the FV discretization scheme for diffusion term. $\mathbf{K}_{p p}^{(2)}$ is expressed as

$$
\mathbf{K}_{p p}^{(2)}=\operatorname{diag}(\mathbf{V} / M)
$$

where $\mathbf{V}$ is a vector for element volume of the mesh.

$$
\mathbf{K}_{p u}=\int_{\Omega}\left(\alpha\left[1-(1-\varphi)^{2}\right]+(1-\varphi)^{2}\right)\left[\mathbf{B}^{u}(1,:)+\mathbf{B}^{u}(2,:)\right]^{T} d \Omega
$$

Therefore $\mathbf{K}_{p u}=-\mathbf{K}_{u p}^{T}$

$$
\mathbf{F}_{p}=\mathbf{Q} d t
$$


Table 1. Algorithm of the numerical program

Initial condition for phase field, displacement field and pressure field

Repeat

Repeat

Compute the phase field by solving Eqn. (35b)

Compute the displacement and pressure field with current phase field by solving Eqn. (50) using the Newton-Raphson method

Until the phase field converges

Until the total simulation time reaches the preset time limit

\subsection{Coupled solution scheme}

There are different ways to solve the three field coupled problem. Considering the strongly coupled relationship between displacement and pressure field, these two fields are solved in a monolithic way while the phase field is solved in a staggered way in this work. The algorithm of the numerical scheme is given in Table 1.

Combining Eqn. (35a) and Eqn. (40) or Eqn. (45) leads to the coupled equation of displacement and pressure field

$$
\left[\begin{array}{ll}
\mathbf{K}_{u u} & \mathbf{K}_{u p} \\
\mathbf{K}_{p u} & \mathbf{K}_{p p}
\end{array}\right]\left[\begin{array}{l}
\mathbf{u} \\
\mathbf{p}
\end{array}\right]-\left[\begin{array}{cc}
0 & 0 \\
\mathbf{K}_{p u} & \mathbf{K}_{p p}^{(2)}
\end{array}\right]\left[\begin{array}{l}
\mathbf{u} \\
\mathbf{p}
\end{array}\right]^{(n)}=\left[\begin{array}{l}
\mathbf{F}_{u} \\
\mathbf{F}_{p}
\end{array}\right]
$$

where $\mathbf{K}_{p p}=\mathbf{K}_{p p}^{(1)}+\mathbf{K}_{p p}^{(2)}$

The solution scheme for solving displacement and pressure field at the $(k+1)$ th Newton-Rapson step based on the $k$ th Newton-Raphson step is expressed as

$$
\left[\begin{array}{l}
\mathbf{u} \\
\mathbf{p}
\end{array}\right]^{(k+1)}=\left[\begin{array}{l}
\mathbf{u} \\
\mathbf{p}
\end{array}\right]^{(k)}-\left[\begin{array}{l}
\mathbf{R}_{u} \\
\mathbf{R}_{p}
\end{array}\right]^{(k)} /\left[\begin{array}{ll}
\mathbf{K}_{u u} & \mathbf{K}_{u p} \\
\mathbf{K}_{p u} & \mathbf{K}_{p p}
\end{array}\right]
$$

where

$$
\left\{\begin{array}{l}
\mathbf{R}_{u}=\mathbf{K}_{u u} \mathbf{u}+\mathbf{K}_{u p} \mathbf{p}-\mathbf{F}_{u} \\
\mathbf{R}_{p}=\mathbf{K}_{p u} \mathbf{u}+\mathbf{K}_{p p} \mathbf{p}-\mathbf{K}_{p u} \mathbf{u}^{(n)}-\mathbf{K}_{p p}^{(2)} \mathbf{p}^{(n)}-\mathbf{F}_{p}
\end{array}\right.
$$

\section{Numerical examples}

Including comparisons with existing phase-field hydraulic fracturing models, a number of test cases are presented in this section to examine the performance of the proposed model. Specifically, § 4.1 considers a single edge notched plate under tension or pure shear loading to validate the accuracy of capturing brittle fractures. $\S 4.2$ considers the deformation of a 1D poroelastic column. It compares the performance of existing and proposed fluid models for capturing the fluid effect in hydraulic fracturing and also demonstrates the superior stability of the proposed hybrid solver in solving the coupled solid deformation and fluid diffusion with the 1D Terzaghi's consolidation problem. $\S 4.3$ verifies the proposed hydraulic fracturing model with the classic semi-analytical solution of hydraulic fracturing in toughness-dominated regime. $§ 4.4$ compares the different injection schemes for pure FEM solver and FE-FV solver and demonstrates the superior performance of the FE-FV solver in mesh and time step convergence. Finally, $\S 4.5$ simulates the propagation of hydraulic fracturing in a naturally fractured formation to demonstrate the capacity of the proposed model in capturing sharp fracture path in complex situations.

\subsection{Single edge notched tension and pure shear tests}

The single edge notched tension and pure shear tests have been extensively used as benchmarks to validate the phase-field fracture model in literatures [23, 31,32]. The geometry and boundary conditions of the tests are depicted in Figure 2. The squared plate has a dimension of $1 \mathrm{~mm}$ by $1 \mathrm{~mm}$ and has a horizontal notch at the middle height 

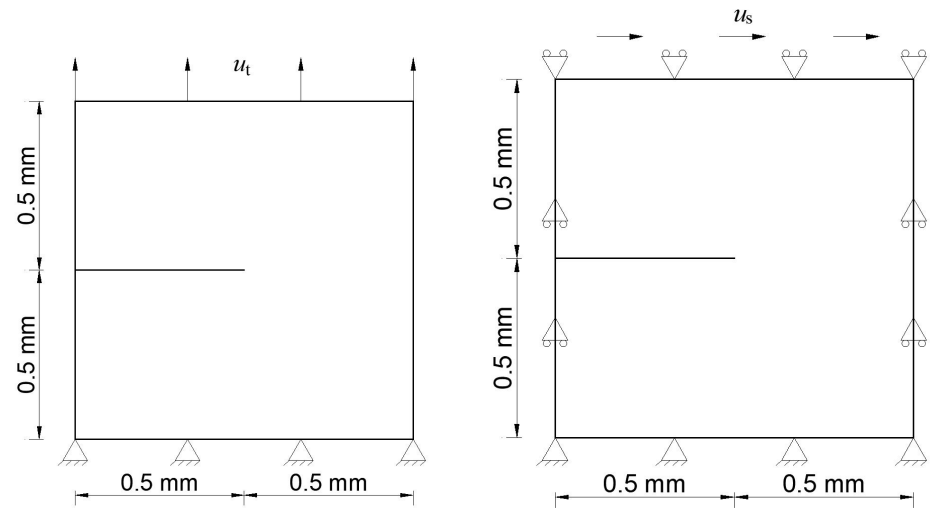

Figure 2. Geometry and boundary conditions for the tension (left) and pure shear (right) tests of a single edge notched square plate
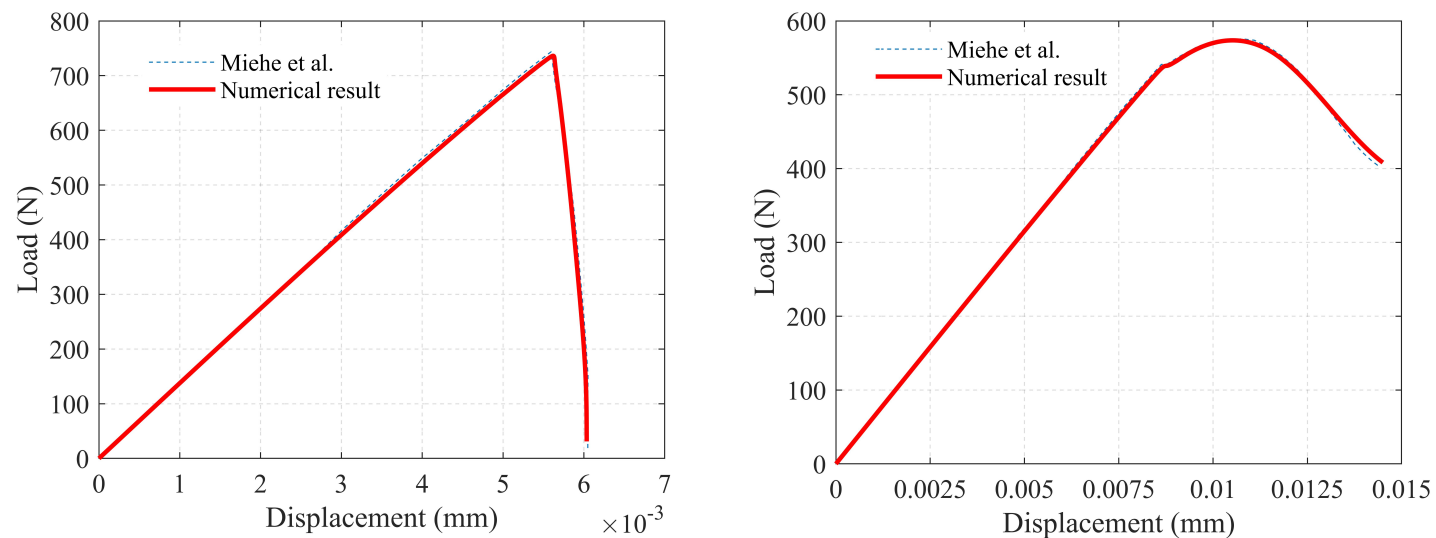

Figure 3. Comparison of load-displacement curves for the single edge notched tension (left) and pure shear (right) tests. The vertical and horizontal loads at the top boundary are used in the tension and pure shear tests respectively. The red solid line indicates our results, and the blue dashed line indicates the literature results [23].

from the left outer surface to the centre. The material parameters are Lamé constant $\lambda=121.15 \mathrm{GPa}$, shear modulus $\mu=80.77 \mathrm{GPa}$ and critical energy release rate $G_{c}=2700 \mathrm{~N} / \mathrm{m}$. In the tension test, the bottom boundary is fixed and a vertical displacement is applied at the top boundary. The displacement increment is $1 \mathrm{e}-5 \mathrm{~mm}$ in the first 500 time steps and is then reduced to 1e- $6 \mathrm{~mm}$ to capture the fracture propagation accurately. In the pure shear test, the bottom boundary is fixed and a shear displacement is applied at the top boundary. In the meantime, the vertical displacement on the left and right boundaries are restricted. A constant displacement increment $1 \mathrm{e}-5 \mathrm{~mm}$ is applied. In both tests, no restriction is applied on the notch. Compared with the results in [23], the phase field is only solved once in each step. The minimum mesh size is set to be $1 \mathrm{e}-3 \mathrm{~mm}$ and $2 \mathrm{e}-3 \mathrm{~mm}$ in the tension and pure shear tests respectively, which is achieved by using a structured mesh of $1000 \times 1000$ and $500 \times 500$ respectively. The length scale parameter $\eta$ is set to be $7.5 \mathrm{e}-3 \mathrm{~mm}$. As shown in Figure 3, the numerical results match well with the literature results.

\subsection{Deformation of a $1 D$ poroelastic column}

\subsubsection{Comparison between different models for capturing the fluid effects}

To accurately compare the performance of Bourdin's model Eqn. (14), Wheeler's model Eqn. (18), Miehe's model Eqn. (21) and the proposed model Eqn. (24) for capturing the fluid effect in hydraulic fracturing, a 1D test case (see Figure 4 and Figure 5) first reported by [33] is adopted. The 1D poroelastic column simulates a part of the formation below the hydraulic fracture. In this case, the displacement of the top surface can be equivalent to the half fracture width. The geometry and boundary conditions of the poroelastic column are illustrated in Figure 5, and the material 


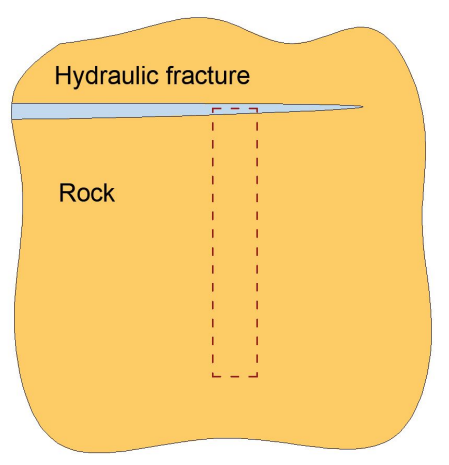

Figure 4. A simplified 1D model for estimating the poroelastic effect during hydraulic fracturing

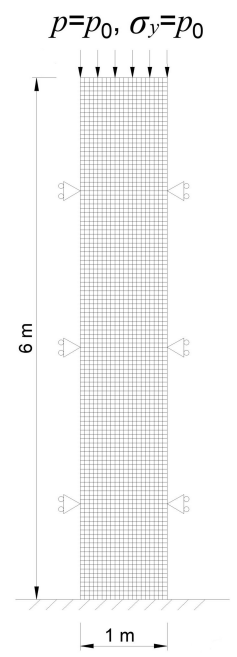

Figure 5. Deformation of a poroelastic column: geometry and boundary conditions

properties are listed in Table $2[34,35]$. The column is fixed at its base and the horizontal displacement on the left and right boundaries are restricted. The left, right and bottom boundaries are all impermeable. Initially, an instantaneous external stress and pore pressure $p_{0}=1 \mathrm{MPa}$ are applied on the top of the column. The numerical model contains 20 and 120 quadrilateral elements along the width and height directions respectively. The time step is set to $0.2 \mathrm{~s}$ in the first $20 \mathrm{~s}$ and gradually increases to $2 \mathrm{~s}$ afterwards. The simulation lasts for $800 \mathrm{~s}$ to reach a steady state condition. The problem is solved with FEM and the corresponding results are consistent with the results in [34], which are used as references to examine the accuracy of different phase field models.

To solve the deformation of poroelastic column using the phase field method, an equivalent boundary condition is introduced. The instantaneous external stress and pore pressure $p_{0}$ applied on the top surface of the poroelastic column is replaced by a fluid pressure $p_{0}$ inside a fracture presented with phase field, as shown by the blue part in Figure 6. In the meantime, the displacement of the top boundary is fixed so that the fracture width in Figure 6 is equivalent to the vertical displacement of the top surface in Figure 5. All four phase field models follow the same form of the total energy (Eqn. (22)), elastic strain energy (Eqn. (23)) and fracture energy (Eqn. (25)). But the fluid effect in Bourdin's model, Wheeler's model, Miehe's model and the proposed model is computed with Eqn. (14), Eqn. (18), Eqn. (21) and Eqn. (24) respectively. For a fair comparison, the pure FE discretization is adopted in all four phase field models to solve the displacement and fluid pressure.

The poroelastic column is solved using the aforementioned five methods. Figure 7 shows the vertical displacement for FEM model or fracture width for phase field models based on different fluid models and the fluid pressure at 
Table 2. Material properties for numerical cases

\begin{tabular}{llllll}
\hline Properties & & Sect. 4.2 & Sect. 4.3 & Sect. 4.4 & Sect. 4.5 \\
Young's modulus $(\mathrm{GPa})$ & $E$ & 14.40 & 17 & 17 & 17 \\
Shear modulus $(\mathrm{GPa})$ & $G$ & 6 & 7.08 & 7.08 & 7.08 \\
Poisson's ratio & $v$ & 0.20 & 0.20 & 0.20 & 0.20 \\
Biot coefficient & $\alpha$ & 0.79 & 0 & 1.0 & 1.0 \\
Biot modulus $(\mathrm{GPa})$ & $M$ & 12.50 & $\mathrm{~N} / \mathrm{A}$ & 12.50 & 12.50 \\
Rock toughness $\left(\mathrm{MPa} \cdot \mathrm{m}^{1 / 2}\right)$ & $K_{I c}$ & $\mathrm{~N} / \mathrm{A}$ & 1.46 & 2 & 2 \\
Intrinsic permeability $\left(\mathrm{m}^{2}\right)$ & $k_{r}$ & $2 \mathrm{e}-14$ & $2 \mathrm{e}-15$ & $2 \mathrm{e}-14$ & $2 \mathrm{e}-15$ \\
Fluid viscosity $(\mathrm{Pa} \cdot \mathrm{s})$ & $\mu$ & $1 \mathrm{e}-3$ & $1 \mathrm{e}-6$ & $1 \mathrm{e}-3$ & $1 \mathrm{e}-3$ \\
\hline
\end{tabular}

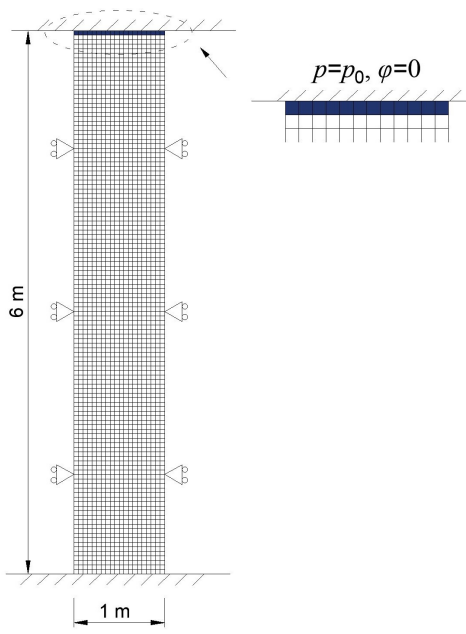

Figure 6. Boundary condition of the poroelastic column using the phase field model: a fracture presented by phase field $\varphi=0$ is inserted into the first row of mesh (blue part) and the top boundary is fixed. A fluid pressure $p_{0}$ is assumed inside the fracture to simulate the instantaneous external stress and pore pressure boundary condition

the bottom are plotted. Miehe's model always underestimates the displacement with a small error for early-time solution and with a high error for late-time solution since the work done by the fluid pressure inside fracture is always underestimated for $\alpha<1$. The results from other four methods agree well with each other for most of the deformation history, except for the initial stage. As shown by the enlarged view in Figure 7, Bourdin's model and Wheerler's model differ significantly from the reference FEM solution for the early-time evolution, while the proposed phase field model agrees well with the reference solution for the entire evolution history. The previous phase field models fail to capture the fluid effect when the fluid pressure is restricted in the area close to the fracture, which corresponds to the case of low permeability reservoir or early stage of hydraulic fracturing.

\subsubsection{Performance of $F E$ and $F V$ solvers in numerical stability}

It has long been recognized that the solution of the coupled solid deformation and fluid diffusion may suffer stability issues, especially in such cases as low permeability or fast loading rates [36, 32]. The non-physical pressure oscillation in phase-field hydraulic fracturing simulation was observed in [19]. The well-known LadyzhenskayaBabuška-Brezzi (LBB) condition was used to check whether or not a specific discretization could guarantee the stability of solution. A higher-order interpolation is normally needed for the displacement field than the pressure field, as in Taylor-Hood elements, which increases the computational cost. Anther option is to introduce additional stability terms in the simulation, for example, [36]. In our model, the LBB condition is automatically satisfied by 

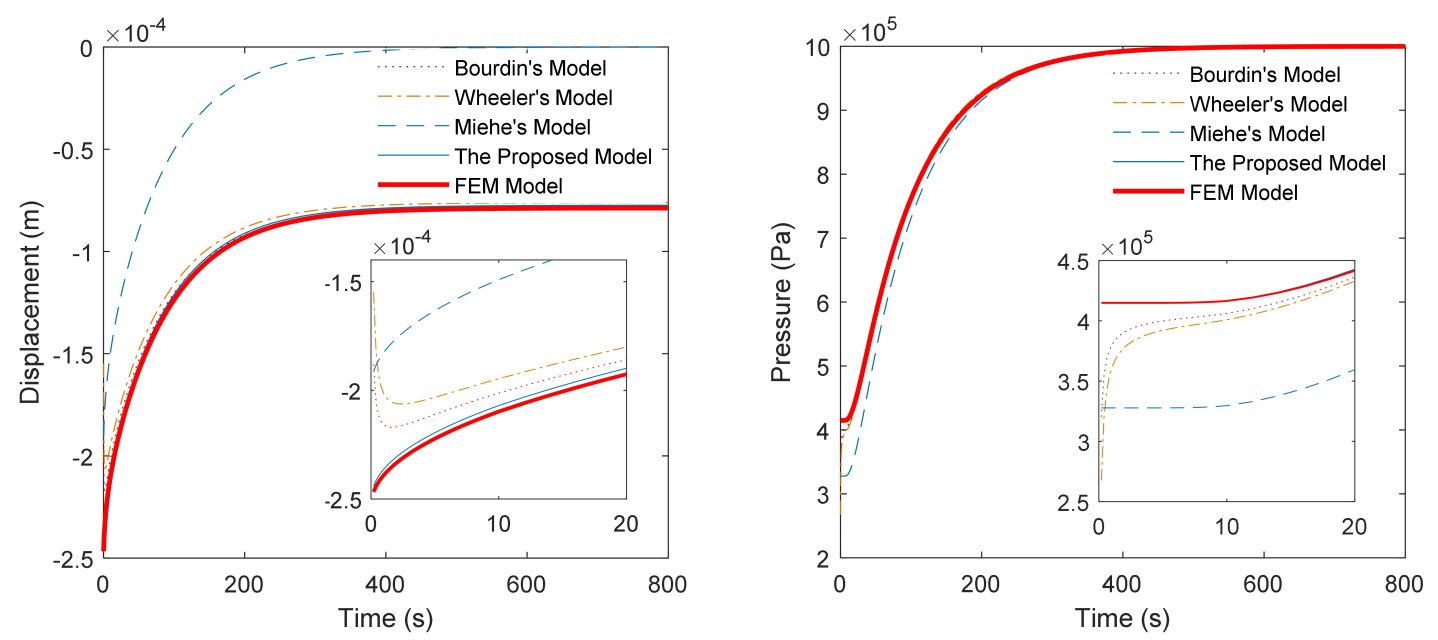

Figure 7. Evolution of the fracture width in phase field model (vertical displacement in FEM model at the top) (left) and the fluid pressure at the bottom (right)

using the FE-FV solution scheme and the stability issue is avoided, which is proved in the following numerical case.

The 1D Terzaghi's consolidation problem, which has a similar formulation with the problem in Figure 5 has been popularly used for checking the stability of different numerical schemes [36, 27, 32]. By setting the pore pressure at the top boundary to zero (i.e. $p=0$ ) and keeping $\sigma_{y}=p_{0}$ and other boundary conditions, the test case in Figure 5 transforms into the Terzaghi's problem as described in [37,32]. The properties of the solid and fluid are kept the same. A relatively large mesh size $(0.5 \mathrm{~m})$ is used in this case to show the difference more clearly. Figure 8 compares the pore pressure distribution along the column obtained by the analytical solution, the numerical solutions from the pure FEM solver with bilinear quadrilateral elements, and the FE-FV solver. The explicit form of the analytical solution is given in Appendix A. The comparison shows that the pore pressure solved by the pure FEM solver contains significant non-physical oscillation while the solution from FE-FV solver is very stable.

\subsection{Validation with semi-analytical solution for toughness-dominated regime}

To prove the accuracy of the proposed model, the toughness-dominated propagation of hydraulic fracture is considered. Due to symmetry, the simulation is based on a half model as shown in Figure 9. The size of the half model is set to be $90 \mathrm{~m}$ by $120 \mathrm{~m}+\mathrm{h}$ to approximate the infinite domain ( $\mathrm{h}$ is the mesh size). The top, bottom and right sides of the model are mechanically restrained and the pressure is set as zero, while a symmetric boundary condition is applied along the left side. The simulation started from an initial fracture with a half-length of $2.2 \mathrm{~m}$ and zero fracture width.

The hydraulic fracture is assumed to propagate in the toughness-dominated regime when the dimensionless viscosity approaches zero. The dimensionless viscosity is expressed as

$$
\mathcal{M}=\frac{\mu^{\prime} Q}{E^{\prime}}\left(\frac{E^{\prime}}{K^{\prime}}\right)^{4}
$$

where

$$
E^{\prime}=\frac{E}{1-v^{2}}, \mu^{\prime}=12 \mu, K^{\prime}=4 \sqrt{\frac{2}{\pi}} K_{I c}
$$

To approximate the limiting case of toughness-dominated regime, a very low fluid viscosity is adopted, as shown in Table 2. The total injection flow rate is $\mathrm{Q}=1 \mathrm{e}-3 \mathrm{~m}^{2} / \mathrm{s}\left(5 \mathrm{e}-4 \mathrm{~m}^{2} / \mathrm{s}\right.$ for the half model). The parameters correspond to $\mathcal{M}=1.43 \mathrm{e}-4$. In the toughness-dominated propagation regime, the leak-off of the fracturing fluid into the reservoir is zero. To simulate this accurately in our model, the leak-off is avoided by only simulating the fluid flow along the horizontal central line and the intrinsic permeability of the elements along the central line is set to be a very low value 


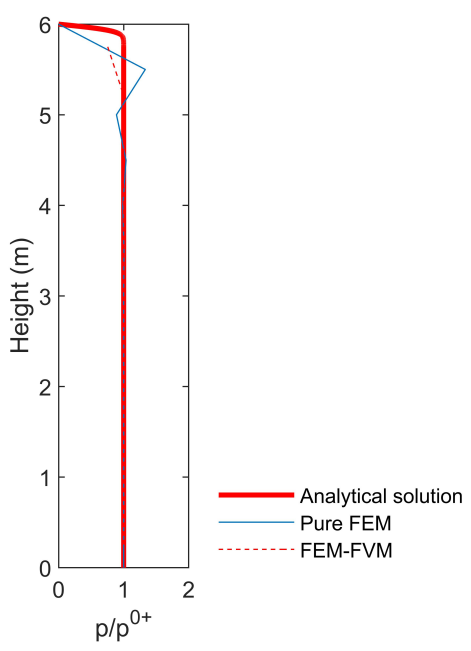

Figure 8. Comparison of the analytical solution, numerical solutions from pure FEM solver and the FE-FV solver for pressure distribution after the first time step $\Delta t=0.01 \mathrm{~s}$. $p^{0+}$ is the pore pressure in the column at $\mathrm{t}=0^{+}$. The pressure results solved by the pure FEM and the FE-FV solver are extracted from the nodes and the element centers respectively.

$\left(2 \mathrm{e}-15 \mathrm{~m}^{2}\right)$. The Biot coefficient is set to zero to approximate the linear elastic condition. FEM is adopted for solving the displacement and phase field while FVM is adopted to discretize the fluid pressure. A tolerance value of 1e-4 is used to stop the Newton-Raphson for displacement and pressure field and the outer loop for phase field.

The evolution of the fracture half-length, fracture width at injection and injection pressure in time are plotted and compared with the analytical solution [38] in Figure 10. It was observed in Santillan et al. [39] that the fracture width directly computed from the displacement field was significantly larger than the analytical value due to the mathematical regularization in phase field model. So they adopted an integral equation of phase field and displacement field to calculate the fracture width. The drawback of treating fracture width as a nonlocal value is the determination of the integral path, especially in complex conditions and extra numerical technique is needed, such as the one proposed by Chukwudozie et al. [30]. In addition, the result is also sensitive to the length scale chosen so a suitable value of length scale needs to be determined in advance. However, the fracture width computed directly from the displacement field in our model shows fairly good comparison between the numerical result and analytical solution and converges to the analytical solution with the decrease of the mesh size and time step. The net pressure at injection point is computed according to the difference between the injection pressure and half of the vertical stress on the left end of the top or bottom boundary (approximation of uniform far-field pressure). The fracture length computed according to Eqn. (4) is slightly larger than the analytical solution due to the phase field distribution around the crack tip.

\subsection{Convergence of FE and FV fluid solvers}

This example considers the propagation of a hydraulic fracture in a porous medium to check the convergence of FEM and FVM fluid solvers with the half model in Figure 9. But the half-length of the initial fracture is changed to 4 $\mathrm{m}$. The material properties are listed in Table 2 . A constant flow of $1 \mathrm{e}-3 \mathrm{~m}^{2} / \mathrm{s}$ is injected into the initial fracture (5e-4 $\mathrm{m}^{2} / \mathrm{s}$ for the half model).

In this numerical case, three different implementations of initial fracture and injection schemes are considered in the framework of FEM or FE-FV, as shown in Figure 11. For the FEM-A scheme, the fluid injection is uniform along the line representing initial fracture, while for the FEM-B and FE-FV schemes, the fluid injections are uniform along the mesh cells representing the initial fracture. The fluid source represented by solid blue dots are computed according to the integral along the line in the FEM-A scheme or over the mesh cells in the FEM-B and FE-FV schemes.

In order to compare the accuracy and convergence of the results computed from the three different injection schemes, three sets of simulation parameters are used: 1) $h=0.4 \mathrm{~m}, \Delta t=0.2 \mathrm{~s}$;2) $h=0.2 \mathrm{~m}, \Delta t=0.1 \mathrm{~s}$ and 3) $h=0.1 \mathrm{~m}, \Delta t=0.05 \mathrm{~s}$ with the length scale parameter $\eta=2 h$. The maximum fluid pressure along the initial 


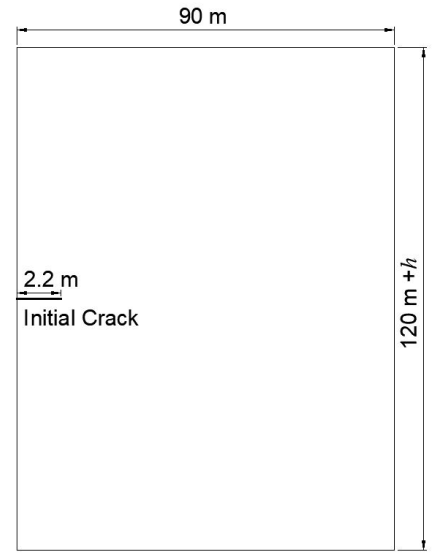

Figure 9. The half model for hydraulic fracturing simulation. The half length of the initial crack is $2.2 \mathrm{~m}$. The geometry of the half model is $90 \mathrm{~m}$ by $120 \mathrm{~m}+h$ to approximate infinite domain, $h$ is the mesh size
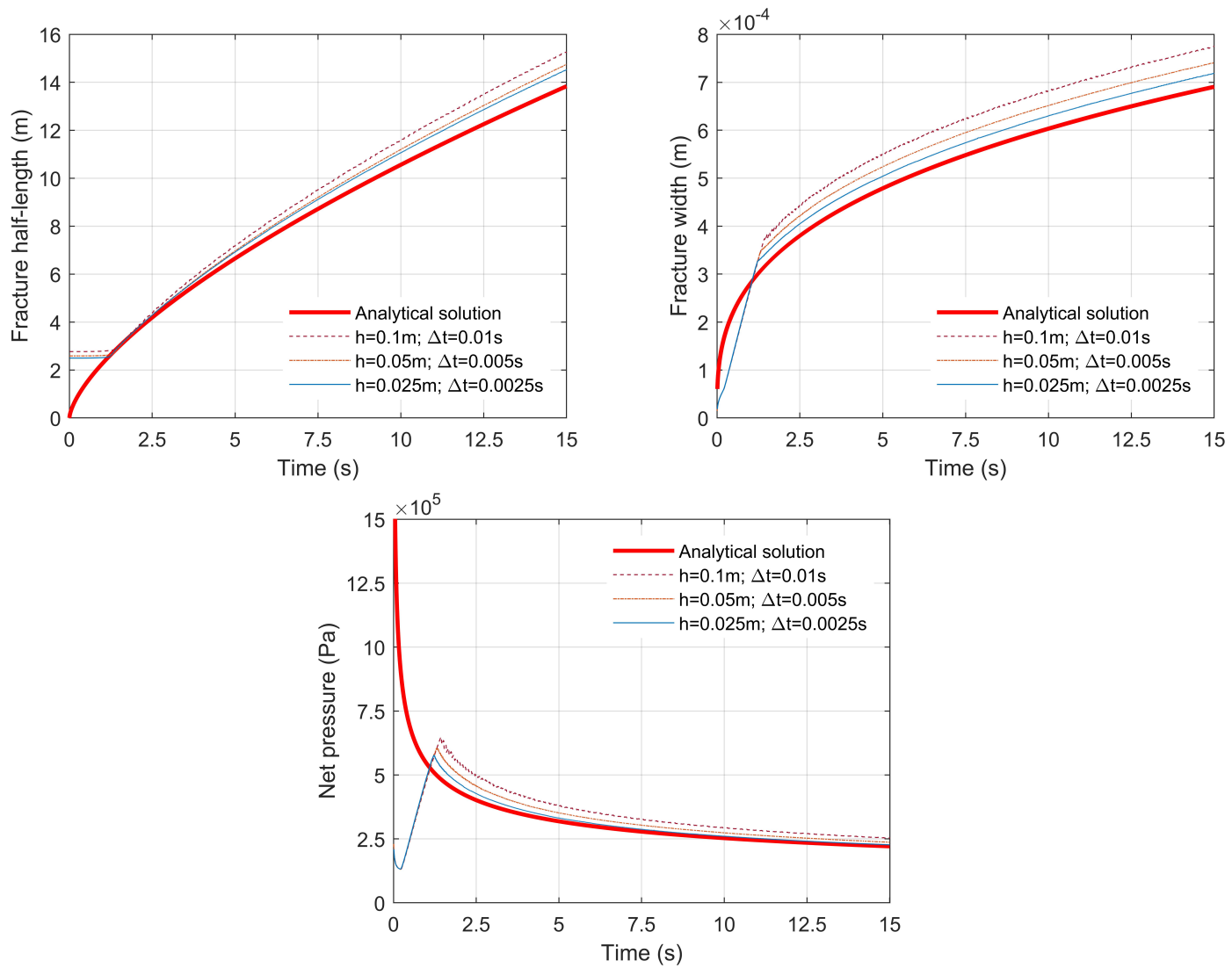

Figure 10. Convergence of the fracture half-length (top left), the fracture width (top right) and the net pressure (bottom) at injection to the analytical solution of toughness-dominated regime with the decrease of mesh size $h$. The length scale is $\eta=0.4 \mathrm{~m}$ and time step is $\Delta t=0.01 \mathrm{~s}$ 

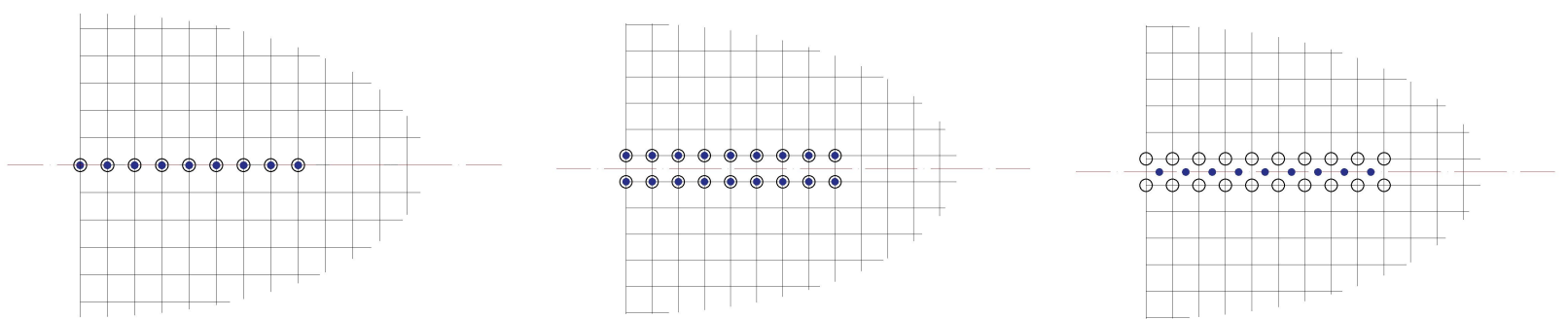

Figure 11. Three different implementations of initial fracture and injection schemes: FEM-A (left), FEM-B (middle) and FE-FV (right). The solid blue dots indicate the fluid source while the open black circles represent the zero phase field.
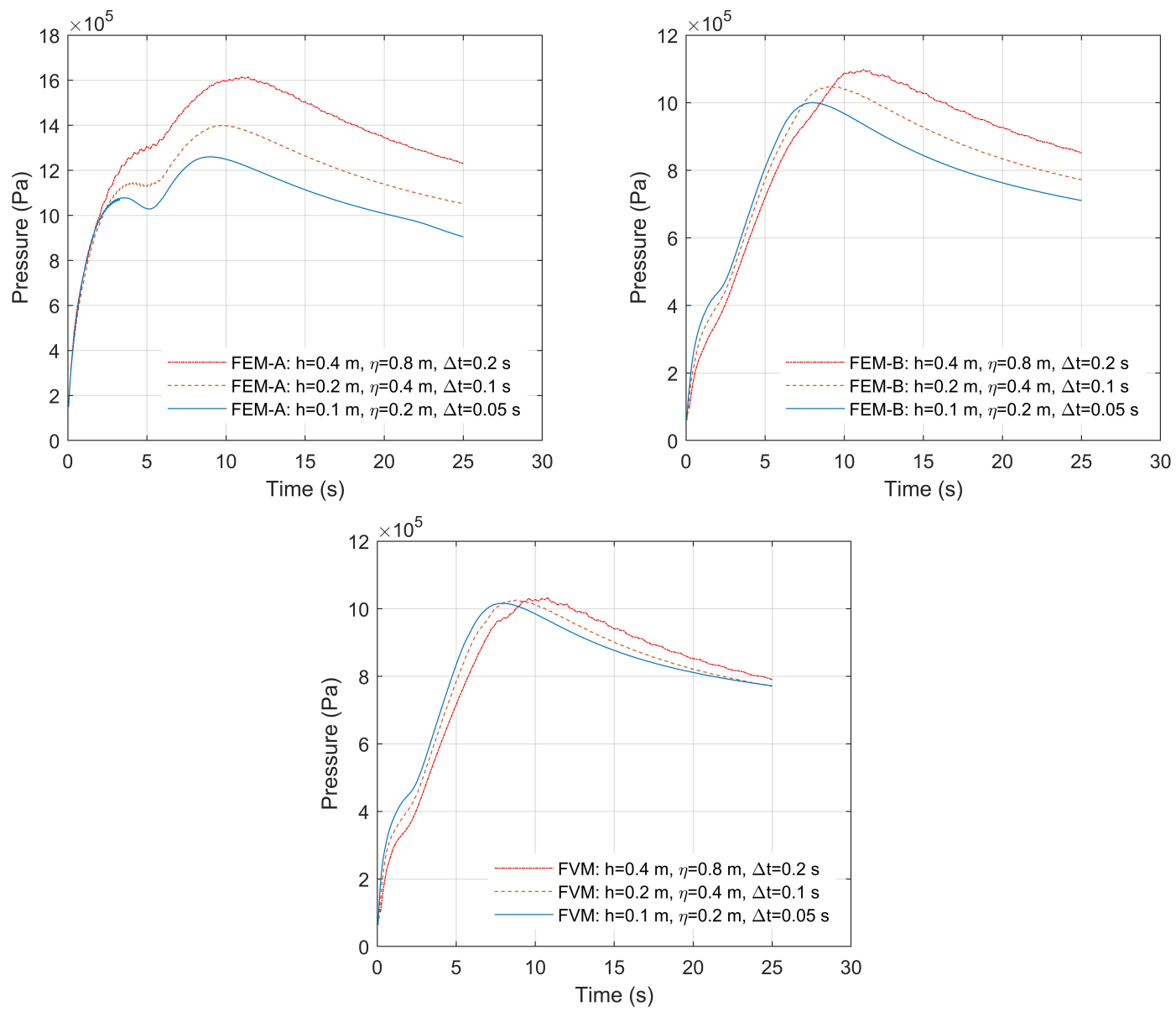

Figure 12. The maximum injection fluid pressure for three different injection schemes: FEM-A (top left), FEM-B (top right) and FE-FV (bottom) 

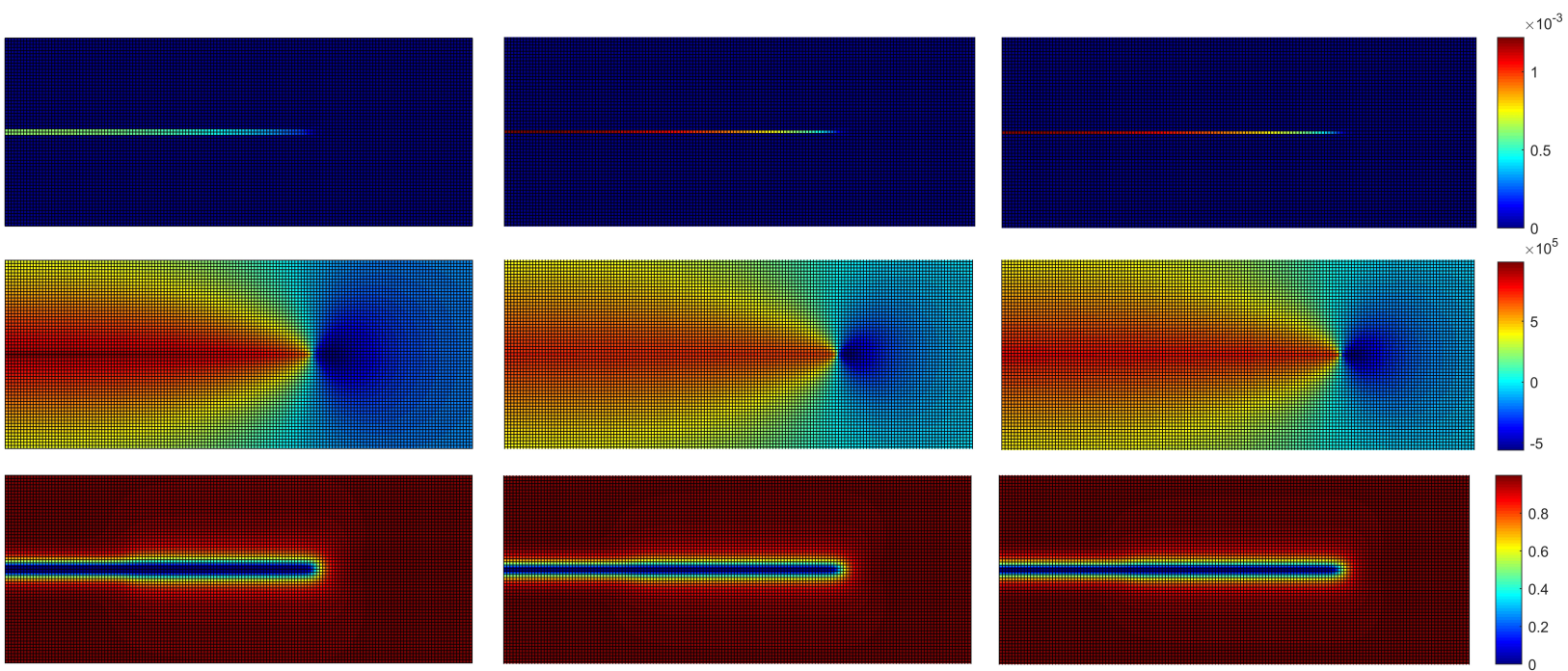

Figure 13. Comparison of the fracture width (top), fluid pressure (middle) and phase field (bottom) at $t=25 \mathrm{~s}$ for three different injection schemes with mesh size $0.1 \mathrm{~m}$ and time step $0.05 \mathrm{~s}$ : FEM-A (left), FEM-B (middle) and FE-FV (right)

fracture is compared in Figure 12. With the decrease of mesh size and time step, all three schemes show a trend of convergence. The accuracy and convergence of the FE-FV scheme is much better than FEM-A scheme and is slightly better than FEM-B schemes.

Demonstrated by this example, FEM-A scheme used in the literature suffers from several drawbacks. First, the FEM-A scheme represents the fracture with two neighbouring rows of mesh cells as shown in Figure 13, but the width of an induced fracture is normally much smaller than the mesh size and therefore should be restricted within one row of mesh cells, as in the FEM-B and FE-FV schemes. The smeared fracture width predicted in the numerical simulation in [29] is not observed. Secondly, the FEM-A scheme significantly overestimates the injection pressure since the initial fracture is not correctly implemented.

\subsection{Propagation of hydraulic fracture in naturally fractured formation}

This example simulates the propagation of hydraulic fracture in a naturally fractured formation, as shown in Figure 14. The natural fracture network is adapted from [40] and is a part of the outcrop pattern mapped at field by Belayneh et al. [41]. An initial fracture with a length of $8 \mathrm{~m}$ is inserted into the center of the model and a constant flow of $1 \mathrm{e}-3 \mathrm{~m}^{2} / \mathrm{s}$ is applied. A structured mesh with a mesh size $h=0.25 \mathrm{~m}$ and time step $\Delta t=0.1 \mathrm{~s}$ are used in the simulation. The initial fracture and natural fractures are both represented by the phase field, which is set to zero for the corresponding nodes. The injection flow rate is applied on the two mesh cells in the middle of the initial fracture. The material properties are listed in Table 2.

The evolution of the fracture width and stimulated fracture network in time are shown in Figure 15. The different scenarios for interaction between hydraulic fracture and natural fracture, including arrest, penetration, reaction were observed. Eventually, a complex fracture network was stimulated, which proves natural fractures are an important source of the fracture complexity. As shown in Figure 15, the proposed model and solution scheme successfully restricted the fracture width in one mesh along most of the fracture path. The stimulated fracture network was recovered according to the fracture width.

\section{Conclusions}

As an promising approach for hydraulic fracturing simulation, phase field model has significant advantages in dealing with complex intersections between hydraulic and natural fractures. In this paper, various fluid models used in phase-field simulation of hydraulic fracturing are reviewed and a revised fluid model is proposed. As proved in the numerical case, the Bourdin's model and Wheeler's model underestimate the fracture width when the fluid pressure is 


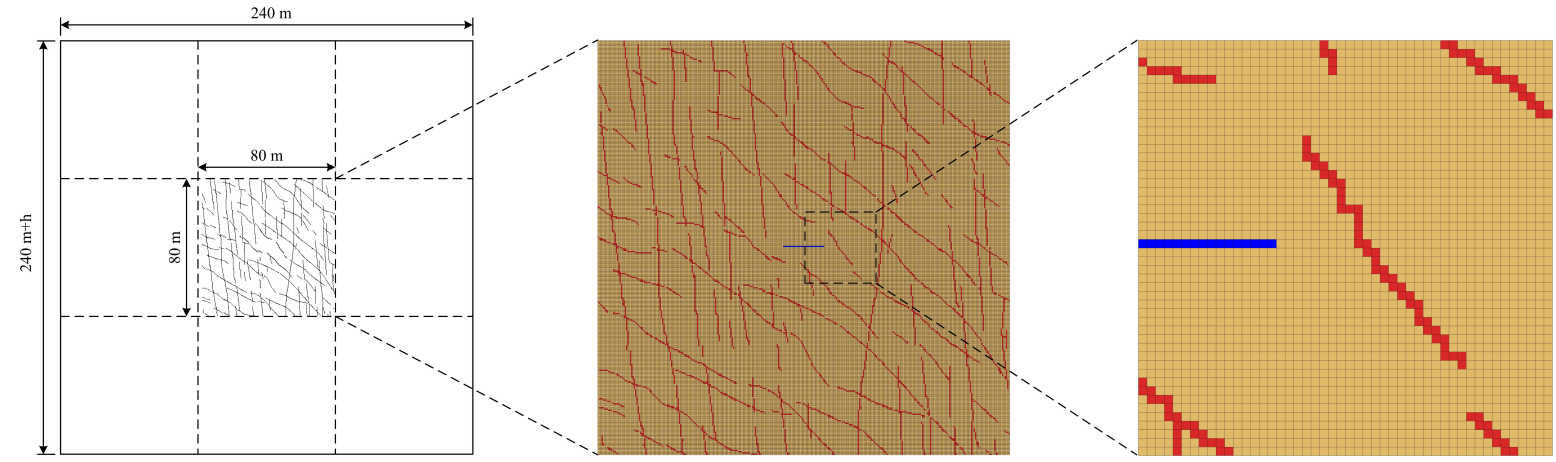

Figure 14. Numerical model for propagation of hydraulic fracture in naturally fractured formation. To approximate the infinite domain, the model of the naturally fractured formation with a size of $80 \mathrm{~m}$ by $80 \mathrm{~m}+\mathrm{h}$ ( $\mathrm{h}$ is the mesh size) is placed at the centrer of a larger model ( $240 \mathrm{~m}$ by 240 $\mathrm{m}+\mathrm{h}$ ). The natural fractures are presented by red solid lines with a thickness of one mesh size and the initial hydraulic fracture in the centre is represented by the blue solid line.
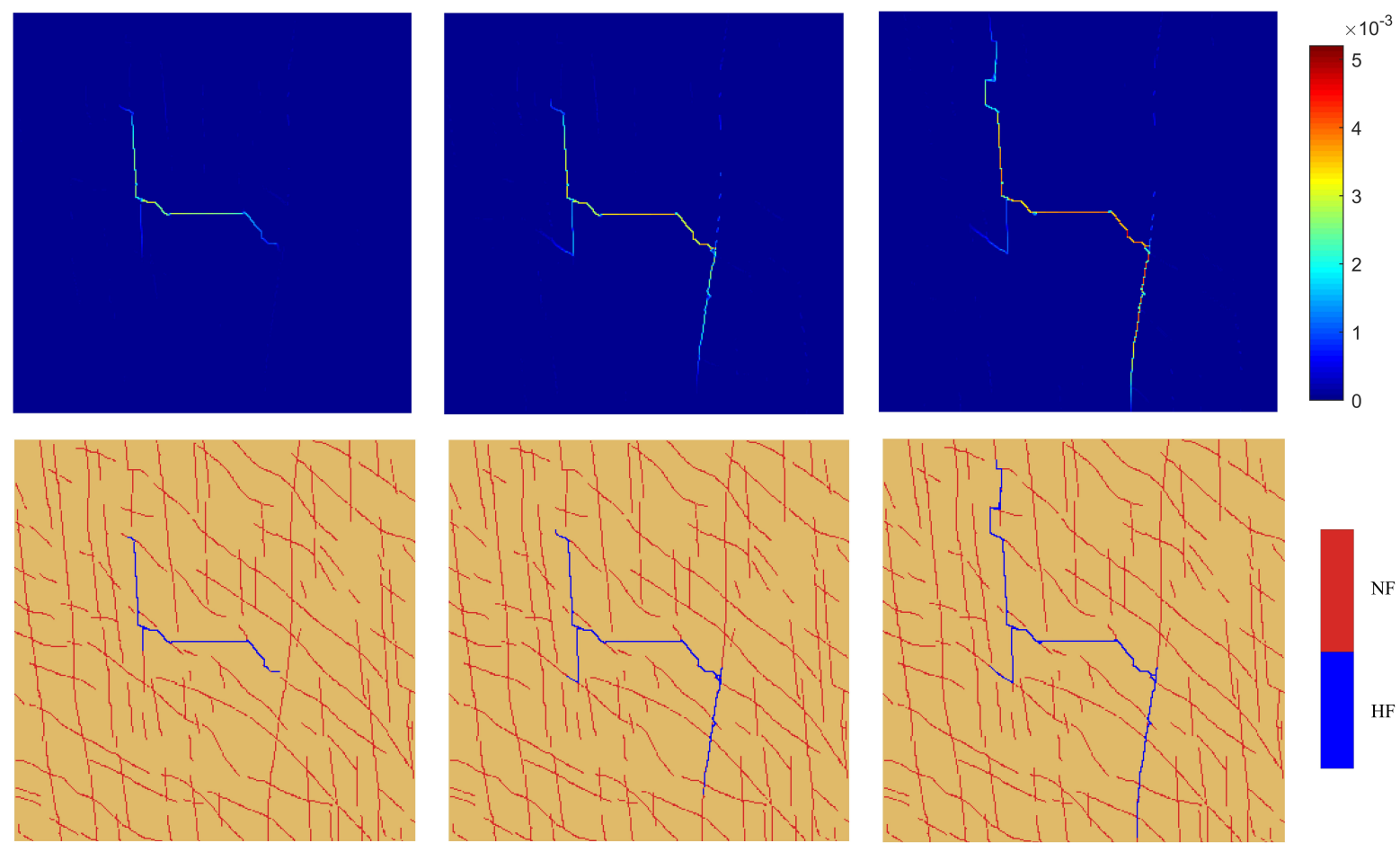

Figure 15. Evolution of the fracture width (top) and stimulated fracture network (bottom) in time: $t=120 \mathrm{~s}$ (left), $\mathrm{t}=240 \mathrm{~s}$ (middle) and $\mathrm{t}=360 \mathrm{~s}$ (right). NF and HF represent the natural fracture and the hydraulic fracture respectively. 
restricted in the area close to the fracture area, which corresponds to the case of low permeability reservoir or earlytime stage of hydraulic fracturing. For biot coefficient $\alpha<1$, Miehe's model always underestimate the fracture width since the work done by the fluid pressure inside the fracture is underestimated, especially when the pore pressure in the reservoir is approaching fluid pressure inside fracture. The proposed model always predicts the fluid effect accurately regardless of the distribution of the fluid pressure over the domain.

In addition, an improved phase-field hydraulic fracturing model with a new FE-FV scheme is implemented and validated. Specifically, the FEM is used to discretize the displacement field and phase field while the FVM for the fluid pressure field. The benefits of adopting FVM for fluid pressure discretization include: 1) the LBB condition is satisfied with the FE-FV solver, which avoids the non-physical oscillation of fluid pressure in the traditional pure FEM solver; 2) the FE-FV solver has a superior performance in mesh and time step convergence; 3) the fracture width is more accurately restricted into one mesh, which could be used for tracking the sharp fracture path. The proposed phase-field hydraulic fracturing model was validated with the analytical solution for toughness-dominated propagation of hydraulic fracture, which shows the revised fluid model and hybrid solver can capture the propagation of hydraulic fracture accurately. Finally, the proposed model was applied in simulating complex propagation of hydraulic fracture in naturally fractured formation.

\section{Acknowledgements}

The authors are grateful to the reviewers for their suggestions and expert reviews. The first author would like to thank the support from China Scholarship Council and the Research Fund of The State Key Laboratory of Coal Resources and Safe Mining, CUMT (SKLCRSM19KF004).

\section{Appendix A.}

The pressure distribution in the 1D Terzaghi's consolidation problem is computed according to [42, 37]

$$
\frac{p(t, z)}{p^{0+}}=\frac{4}{\pi} \sum_{j=1}^{\infty} \frac{(-1)^{j-1}}{(2 j-1)} \cos \left[(2 j-1) \frac{\pi}{2}\left(\frac{z}{H}\right)\right] \exp \left[-(2 j-1)^{2} \frac{\pi^{2}}{4} \frac{c_{v} t}{H^{2}}\right]
$$

where $z$ is the vertical coordinate, $H$ is the height of the column. The pore pressure in the column at $\mathrm{t}=0^{+}$and coefficient of consolidation are expressed as

$$
\begin{aligned}
p^{0+} & =\frac{\alpha m_{v}}{1 / M+\alpha^{2} m_{v}} p_{0} \\
c_{v} & =\frac{k}{\mu} \frac{1}{1 / M+m_{v}}
\end{aligned}
$$

where $\alpha$ and $M$ are the biot coefficient and biot modulus respectively, $p_{0}$ is the applied force, $k$ is the intrinsic permeability and $\mu$ is the fluid viscosity. The confined compressibility $m_{v}$ is expressed as

$$
m_{v}=\frac{(1+v)(1-2 v)}{(1-v) E}
$$

\section{References}

[1] J. Adachi, E. Siebrits, A. Peirce, J. Desroches, Computer simulation of hydraulic fractures, International Journal of Rock Mechanics and Mining Sciences 44 (5) (2007) 739-757. doi:https://doi.org/10.1016/j.ijrmms.2006.11.006.

[2] S. Secchi, B. A. Schrefler, A method for 3-d hydraulic fracturing simulation, International Journal of Fracture 178 (1-2) (2012) $245-258$. doi:http://dx.doi.org/10.1007/s10704-012-9742-y.

[3] B. Chen, A. R. Barron, D. R. J. Owen, C.-F. Li, Propagation of a plane strain hydraulic fracture with a fluid lag in permeable rock, Journal of Applied Mechanics 85 (9) (2018) 091003-091003-10. doi:10.1115/1.4040331.

[4] B. Chen, S. Cen, A. R. Barron, D. R. J. Owen, C.-F. Li, Numerical investigation of the fluid lag during hydraulic fracturing, Engineering Computationsdoi:10.1108/EC-02-2018-0087. 
[5] M. M. Hossain, M. K. Rahman, Numerical simulation of complex fracture growth during tight reservoir stimulation by hydraulic fracturing, Journal of Petroleum Science and Engineering 60 (2) (2008) 86-104. doi:http://dx.doi.org/10.1016/j.petrol.2007.05.007.

[6] T. Wang, Z. Liu, Y. Gao, Q. Zeng, Z. Zhuang, Theoretical and numerical models to predict fracking debonding zone and optimize perforation cluster spacing in layered shale, Journal of Applied Mechanics 85 (1) (2017) 011001. doi:10.1115/1.4038216.

[7] T. Wang, Z. Liu, Q. Zeng, Y. Gao, Z. Zhuang, Xfem modeling of hydraulic fracture in porous rocks with natural fractures, Science China Physics, Mechanics Astronomy 60 (8). doi:10.1007/s11433-017-9037-3.

[8] P. Gupta, C. A. Duarte, Simulation of non-planar three-dimensional hydraulic fracture propagation, International Journal for Numerical and Analytical Methods in Geomechanics 38 (13) (2014) 1397-1430. doi:http://dx.doi.org/10.1002/nag.2305.

[9] L. C. Li, C. A. Tang, G. Li, S. Y. Wang, Z. Z. Liang, Y. B. Zhang, Numerical simulation of 3d hydraulic fracturing based on an improved flowstress-damage model and a parallel fem technique, Rock Mechanics and Rock Engineeringdoi:http://dx.doi.org/10.1007/s00603-012-0252-z.

[10] M. Wangen, Finite element modeling of hydraulic fracturing in 3d, Computational Geosciences 17 (4) (2013) 647-659. doi:http://dx.doi.org/10.1007/s10596-013-9346-2.

[11] M. Profit, M. Dutko, J. Yu, S. Cole, D. Angus, A. Baird, Complementary hydro-mechanical coupled finite/discrete element and microseismic modelling to predict hydraulic fracture propagation in tight shale reservoirs, Computational Particle Mechanics 3 (2) (2016) $229-248$. doi:https://doi.org/10.1007/s40571-015-0081-4.

[12] A. Obeysekara, Numerical modelling of hydraulic fracturing in naturally fractured rock, Thesis (2018).

[13] Q. Zhao, A. Lisjak, O. Mahabadi, Q. Liu, G. Grasselli, Numerical simulation of hydraulic fracturing and associated microseismicity using finite-discrete element method, Journal of Rock Mechanics and Geotechnical Engineering 6 (6) (2014) 574 - 581. doi:https://doi.org/10.1016/j.jrmge.2014.10.003.

[14] G. A. Francfort, J. J. Marigo, Revisiting brittle fracture as an energy minimization problem, Journal of the Mechanics and Physics of Solids 46 (8) (1998) 1319-1342. doi:http://doi.org/10.1016/S0022-5096(98)00034-9.

[15] X. Li, C. Dongyang, G. Yue, L. Zhanli, Numerical study on crack propagation in linear elastic multiphase composite materials using phase field method, Engineering Computations 36 (1) (2018) 307-333. doi:10.1108/EC-03-2018-0116.

[16] B. Bourdin, C. P. Chukwudozie, K. Yoshioka, A variational approach to the numerical simulation of hydraulic fracturing, Vol. SPE-159154MS, Society of Petroleum Engineers, 2012. doi:https://doi.org/10.2118/159154-MS

[17] K. Yoshioka, B. Bourdin, A variational hydraulic fracturing model coupled to a reservoir simulator, International Journal of Rock Mechanics and Mining Sciences 88 (2016) 137-150. doi:http://dx.doi.org/10.1016/j.ijrmms.2016.07.020.

[18] M. F. Wheeler, T. Wick, W. Wollner, An augmented-lagrangian method for the phase-field approach for pressurized fractures, Computer Methods in Applied Mechanics and Engineering 271 (2014) 69-85. doi:10.1016/j.cma.2013.12.005.

[19] S. Mauthe, C. Miehe, Hydraulic fracture in poro-hydro-elastic media, Mechanics Research Communications 80 (2017) 69-83. doi:10.1016/j.mechrescom.2016.09.009.

[20] W. Ehlers, C. Luo, A phase-field approach embedded in the theory of porous media for the description of dynamic hydraulic fracturing, Computer Methods in Applied Mechanics and Engineering 315 (2017) 348-368. doi:10.1016/j.cma.2016.10.045.

[21] M. Hofacker, C. Miehe, A phase field model of dynamic fracture: Robust field updates for the analysis of complex crack patterns, International Journal for Numerical Methods in Engineering 93 (3) (2013) 276-301. doi:10.1002/nme.4387.

[22] H. Amor, J.-J. Marigo, C. Maurini, Regularized formulation of the variational brittle fracture with unilateral contact: Numerical experiments, Journal of the Mechanics and Physics of Solids 57 (8) (2009) 1209-1229. doi:10.1016/j.jmps.2009.04.011.

[23] C. Miehe, M. Hofacker, F. Welschinger, A phase field model for rate-independent crack propagation: Robust algorithmic implementation based on operator splits, Computer Methods in Applied Mechanics and Engineering 199 (45-48) (2010) 2765-2778. doi:10.1016/j.cma.2010.04.011.

[24] T. Wick, G. Singh, M. F. Wheeler, Fluid-filled fracture propagation with a phase-field approach and coupling to a reservoir simulator, SPE Journal 21 (03) (2016) 981-999. doi:10.2118/168597-PA.

[25] S. Lee, M. F. Wheeler, T. Wick, S. Srinivasan, Initialization of phase-field fracture propagation in porous media using probability maps of fracture networks, Mechanics Research Communications 80 (2017) 16-23. doi:10.1016/j.mechrescom.2016.04.002.

[26] S. Lee, M. F. Wheeler, T. Wick, Iterative coupling of flow, geomechanics and adaptive phase-field fracture including level-set crack width approaches, Journal of Computational and Applied Mathematics 314 (2017) 40-60. doi:10.1016/j.cam.2016.10.022.

[27] C. Miehe, S. Mauthe, S. Teichtmeister, Minimization principles for the coupled problem of darcy-biot-type fluid transport in porous media linked to phase field modeling of fracture, Journal of the Mechanics and Physics of Solids 82 (2015) $186-217$. doi:http://doi.org/10.1016/j.jmps.2015.04.006.

[28] C. Miehe, S. Mauthe, Phase field modeling of fracture in multi-physics problems. part iii. crack driving forces in hydro-poro-elasticity and hydraulic fracturing of fluid-saturated porous media, Computer Methods in Applied Mechanics and Engineering 304 (2016) 619-655. doi:http://doi.org/10.1016/j.cma.2015.09.021.

[29] L. Xia, J. Yvonnet, S. Ghabezloo, Phase field modeling of hydraulic fracturing with interfacial damage in highly heterogeneous fluid-saturated porous media, Engineering Fracture Mechanics 186 (2017) 158-180. doi:10.1016/j.engfracmech.2017.10.005.

[30] C. Chukwudozie, B. Bourdin, K. Yoshioka, A variational phase-field model for hydraulic fracturing in porous media, Computer Methods in Applied Mechanics and Engineering 347 (2019) 957 - 982. doi:https://doi.org/10.1016/j.cma.2018.12.037.

[31] G. Liu, Q. Li, M. A. Msekh, Z. Zuo, Abaqus implementation of monolithic and staggered schemes for quasi-static and dynamic fracture phase-field model, Computational Materials Science 121 (2016) 35 - 47. doi:https://doi.org/10.1016/j.commatsci.2016.04.009.

[32] G. Guo, M. Fall, Modelling of preferential gas flow in heterogeneous and saturated bentonite based on phase field method, Computers and Geotechnics 116 (2019) 103206. doi:10.1016/j.compgeo.2019.103206.

[33] J. D. Detournay, E.and McLennan, J. C. Roegiers, Poroelastic concepts explain some of the hydraulic fracturing mechanismsdoi:/10.2118/15262-MS.

[34] T. J. Boone, A. R. Ingraffea, A numerical procedure for simulation of hydraulically-driven fracture propagation in poroelastic media, International Journal for Numerical and Analytical Methods in Geomechanics 14 (1) (1990) 27-47. doi:http://dx.doi.org/10.1002/nag.1610140103.

[35] S. Secchi, L. Simoni, B. A. Schrefler, Mesh adaptation and transfer schemes for discrete fracture propagation in porous materials, International 
Journal for Numerical and Analytical Methods in Geomechanics 31 (2) (2007) 331-345. doi:http://dx.doi.org/10.1002/nag.581.

[36] J. A. White, R. I. Borja, Stabilized low-order finite elements for coupled solid-deformation/fluid-diffusion and their application to fault zone transients, Computer Methods in Applied Mechanics and Engineering 197 (49) (2008) 4353 - 4366. doi:https://doi.org/10.1016/j.cma.2008.05.015.

[37] V. P. Nguyen, H. Lian, T. Rabczuk, S. Bordas, Modelling hydraulic fractures in porous media using flow cohesive interface elements, Engineering Geology 225 (2017) 68 - 82, special Issue: Characterisation of Fractures in Rock: from Theory to Practice (ROCKFRAC). doi:https://doi.org/10.1016/j.enggeo.2017.04.010.

[38] D. I. Garagash, Plane-strain propagation of a fluid-driven fracture during injection and shut-in: Asymptotics of large toughness, Engineering Fracture Mechanics 73 (4) (2006) 456-481. doi:http://doi.org/10.1016/j.engfracmech.2005.07.012.

[39] D. Santillán, R. Juanes, L. Cueto-Felgueroso, Phase field model of fluid-driven fracture in elastic media: Immersed-fracture formulation and validation with analytical solutions, Journal of Geophysical Research: Solid Earth 122 (4) (2017) 2565-2589. doi:10.1002/2016jb013572.

[40] Q. Lei, J.-P. Latham, C.-F. Tsang, J. Xiang, P. Lang, A new approach to upscaling fracture network models while preserving geostatistical and geomechanical characteristics, Journal of Geophysical Research: Solid Earth 120 (7) (2015) 4784-4807. doi:10.1002/2014JB011736.

[41] M. Belayneh, S. Matthai, M. Blunt, S. Rogers, Comparison of deterministic with stochastic fracture models in water-flooding numerical simulations, AAPG Bulletin 93 (2009) 1633-1648. doi:10.1306/07220909031.

[42] K. Terzaghi, Theoretical Soil Mechanics, John Wiley and Sons, Ltd, 1943. 